Chapter 1

\title{
Protocol Requirements for Self-organizing Artifacts: Towards an Ambient Intelligence
}

\author{
Carlos Gershenson and Francis Heylighen \\ Centrum Leo Apostel, Vrije Universiteit Brussel, Belgium \\ $\{$ cgershen,fheyligh\}@vub.ac.be
}

\begin{abstract}
We discuss which properties common-use artifacts should have to collaborate without human intervention. We conceive how devices, such as mobile phones, PDAs, and home appliances, could be seamlessly integrated to provide an "ambient intelligence" that responds to the user's desires without requiring explicit programming or commands. While the hardware and software technology to build such systems already exists, as yet there is no standard protocol that can learn new meanings. We propose the first steps in the development of such a protocol, which would need to be adaptive, extensible, and open to the community, while promoting self-organization. We argue that devices, interacting through "game-like" moves, can learn to agree about how to communicate, with whom to cooperate, and how to delegate and coordinate specialized tasks. Thus, they may evolve a distributed cognition or collective intelligence capable of tackling complex tasks.
\end{abstract}

\subsection{A Scenario}

The diversity and capabilities of devices we use at home, school, or work, are increasing constantly. The functions of different devices often overlap (e.g. a portable computer and a mobile phone have agendas; a radio-clock and a PDA 
have alarms), but most often we cannot combine their capabilities automatically (e.g. the PDA cannot tell the radio to set its alarm for the early Tuesday's appointment), and users need to repeat the same tasks for different devices (e.g. setting up an address book in different devices). Moreover, using the functionality of some devices in combination with others would be convenient (e.g. if my computer has an Intelligent User Interface, I would like to use it to ask for coffee, without the need of having speech recognition in the coffee machine: The computer should be able to ask the coffee machine for cappuccino).

Could we build devices so that they would automatically coordinate, combining their functions, and possibly producing new, "emergent" ones? The technology to achieve this is already at hand. What we lack is a proper design methodology, able to tackle the problems posed by autonomously communicating artifacts in a constantly changing technosphere. In this paper we try to delineate the requirements that such a design paradigm should fulfill. The scenario we imagine considers a nearby future where technological artifacts selforganize, in the sense that they are able to communicate and perform desirable tasks with minimal human intervention.

This vision is closely related to the concept of "Ambient Intelligence" (AmI) 13, which envisages a future where people are surrounded by "smart" and "sensitive" devices. AmI would be the result of the integration of three technologies: Ubiquitous Computing [17, Ubiquitous Communication, and Intelligent User Friendly Interfaces. The first one conceives of a seamless integration of computation processes taking place in the variety of artifacts that surround us, being part of "The Grid", the network that would allow anyone anywhere to access the required computing power. The present paper focuses on the aspect of Ubiquitous Communication that attempts to obtain seamless information exchange between devices. Intelligent User Friendly Interfaces should enable an intuitive, effortless interaction between users and devices.

With current approaches, this scenario would be possible, since we have the technology, but extremely expensive, since people would need to buy from the same producer all of their devices. We can see a similar casein the area of Home Automation: the technology is available on the market, but it is not possible to buy today ventilation for a house, and in five years integrate the system with a new fire detector. An engineer needs to integrate them manually, so that the ventilation system could be activated if smoke is detected, simply because the ventilation system was not designed to receive such signals. These limitations increase the price and restrict the market of devices for Home Automation, since complete solutions should be bought in order to have full coordination and functionality between devices. People would be more willing to invest in Home Automation if they could have the possibility of acquiring it progressively.

\subsection{Requirements for self-organizing artifacts}

We see self-organization as a paradigm for designing, controlling, and understanding systems [6]. A key characteristic of a self-organizing system is that 
structure and function of the system "emerge" from interactions between the elements. The purpose should not be explicitly designed, programmed, or controlled. The components should interact freely with each other and with the environment, mutually adapting to reach an intrinsically "preferable" or "fit" configuration (attractor), thus defining an emergent purpose for the system [10]. By "self-organizing artifacts" we mean a setup where different devices, with different fabrications and functionalities, and moving in and out of different configurations, can communicate and integrate information to produce novel functionalities that the devices by themselves could not achieve.

A first requirement for such communication is cross-platform compatibility. This is already achieved for programming with Java, and for documents with XML. Another requirement is wireless communication, which is offered by technologies such as IR, Bluetooth and WiFi. Near Field Communications (NFC) is a newly envisioned standard, proposed by a consortium headed by Sony, Nokia, and Philips, which would allow information to be transmitted between devices that come in close spatial proximity ("touching").

Even with such a standard, the problem remains that the user generally would need to specifically request such communication between devices (e.g. "transfer this file from here to there"). Ideally, the devices would know what we want them to do and how to do it. User Interfaces already help us to tell them our wishes. Still, one device cannot tell another device what we want, especially if they are produced by different manufacturers. This is a general problem of communication between artifacts: they can recognize standard messages, but they do not "know" what the messages mean. To avoid endless debates, we can say that the meaning of a message is determined by its use [18: if a device has received a message, and does "the right thing" (for the user), then it has "understood" the meaning of the message. Thus, the user's satisfaction is the ultimate measure of the effectiveness of the artifacts' performance.

Another issue is how to deal with changes in technology. We do not want to reconfigure every artifact each time a new device arrives. Moreover, we want the old devices to be able at least to cope with the functionality of new ones. New devices should configure themselves as automatically as possible. Older ones may require user intervention at first (as they cannot know beforehand which functions will be required), but they should be able to cope with new technology being added to the network. The overall system must be adaptive, extensible, and open.

An adaptive system can cope with unexpected changes in its environment, as exemplified by the constantly changing technology. Having flexibility built into our systems is desirable: they should at least be able to tolerate events they were not designed for without breaking down, but preferably try to find adapted solutions, or at least ask assistance from the user. For example, home appliances have a limited set of functions. To have them self-organize (e.g. the alarm clock coordinating with the microwave oven, and the oven with the kettle), their functions could be easily programmed to respond to unknown messages. If a new device arrives, and an old one does not know what to do when it receives 
a message, it can check what the user wants, thus learning how to respond appropriately. The possibility to add more devices to an existing configuration may be called extensibility.

Suppose that a company develops adaptable and extensible devices that interact seamlessly with each other. This would still leave the problem that customers cannot add devices from other companies, as these would follow their own standards, thus creating compatibility problems. We believe that the solution is to have open technologies, in the spirit of GNU. Open means that everyone has free access to their specifications. The advantage is that they can develop much faster, meeting the requirements of more people, because they are developed by a global community that can try out many more approaches than any single company. Still, a company can benefit in promoting an open technology, since this would provide them with free publicity while everyone is using their protocol (e.g. Sun's Java).

Open technology can respond to the needs of the largest variety of people, while allowing problems and errors to be detected and corrected more easily. Another advantage is that it allows people to get updates developed by other users for free. For example, if I program my "old" toaster to integrate with my new mobile phone, it costs me nothing to make the program available on the Internet to anyone else who might need it. Thus, updates, extensions, and specialized applications can flow much more quickly from a global community than from a private company. Still, companies would benefit from this approach, since people would be more willing to buy new devices as integrating them into their existing, open setup, will be easier.

\subsection{Achieving self-organization}

We can divide the problem of self-organizing integration into three subproblems:

1) devices should learn to communicate with each other, even when they have no a priori shared understanding of what a particular message or function means; 2 ) devices should learn which other devices they can trust to cooperate, avoiding the others; 3) devices should develop an efficient division of labour and workflow, so that each performs that part of the overall task that it is most competent at, at the right moment, while delegating the remaining functions to the others.

These issues are all part of collective intelligence [8] or distributed cognition 11]: a complex problem cannot be tackled by a single device or agent, but must be solved by them working together, in an efficiently coordinated, yet spatially distributed, system, where information flows from the one agent to the other according to well-adapted rules. Until now, distributed cognition has been studied mostly in existing systems, such as human organizations [11 or animal "swarms" [3], that have evolved over many generations to develop workable rules. Having the rules self-organize from scratch is a much bigger challenge, which has been addressed to some degree in distributed AI and multi-agent simulations of social systems. Inspired by these first explorations, we will propose a number of general mechanisms that could probably tackle the three subproblems. 
However, extensive simulation will clearly be needed to test and elaborate these mechanisms.

\subsection{Learning to communicate}

To communicate effectively, different agents must use the same concepts or categories. To achieve effective coordination, agents must reach a shared understanding of a concept, so that they agree about which situations and actions belong to that category, and which do not. A group of agents negotiating such a consensus may self-organize, so that a globally shared categorisation emerges out of local interactions between agents.

Such self-organization has been shown in different simulations of the evolution of language [16, 12. Here, interacting software agents or robots try to develop a shared lexicon, so that they interpret the same expressions, symbols, or "words" in the same way. In these simulations agents interact according to a protocol called a "language game". There are many varieties of such games, but the general principle is that two agents "meet" in virtual space, which means that through their sensors they experience the same situation at the same time. Then they try to achieve a consensus on how to designate one of the components of their shared experience by each in turn performing elementary moves.

In a typical move, the first agent produces an "utterance" referring to a phenomenon that belongs to one of its inbuilt or previously learned categories, and the second one finds the best fitting category for that phenomenon in its knowledge base. The second agent then indicates a phenomenon belonging to that same category. If this phenomenon also belongs to the same category for the first agent, both categorisations are reinforced, otherwise they are reduced in strength. In the next move of the "game", another phenomenon is indicated, which may or may not belong to the category. The corresponding categorisation is strengthened or weakened depending on the degree of agreement. After a number of moves the game is stopped, each agent maintaining the mutually adjusted categories. Each agent in turn is coupled to another agent in the system, to play a new game using different phenomena. After some games a stable and coherent system of categories shared by all agents is likely to emerge through self-organization. A good example of such a set-up can be found in Belpaeme's 22 simulation of the origin of shared colour categories.

If for some reason devices are not able to communicate, they should be able to notify the user, and ask for the correct interpretation of the message. This is easy, since devices have a limited functionality. It would be possible to "teach" a device what to do if it receives a particular message, and the device should "learn" the meaning of the message.

Research has been done in multi-agent systems where agents negotiate their protocols 14, 4], which could be extended for a setup of self-organizing artifacts. However, agent communication standards, such as FIPA, still do not contemplate adaptation to new meanings. Nevertheless, there is promising research going on in this direction. 


\subsection{Learning to cooperate}

Integrated devices should not only communicate, but cooperate. Cooperation may seem self-evident in preprogramed systems, where the components are explicitly designed to respond appropriately to requests made by other components. However, this is no longer the case in open, extensible configurations.

For example, a person at the airport would like her PDA to collaborate with the devices present at the airport, so that it can automatically warn her when and where she has to go, or tell her which facilities are available in the airport lounge. Yet not all devices at the airport may be ready to help a PDA, e.g. because of security restrictions, because they are proprietary and reserved for paying customers, or because they simply do not care about personal wishes. Moreover, devices may be ready to share certain types of services but not others, e.g. telling users when the flight is scheduled to depart, but not how many passengers will be on it. As another example, devices may not only be uncooperative, but malevolent, in the sense that they try to manipulate other devices in a way detrimental to their user. Such devices may be programmed, e.g. by fraudsters, spies, or terrorists.

There exists an extensive literature on the evolution of cooperation between initially "selfish" agents, inspired by the seminal work of Axelrod [1] that compared different strategies for playing a repeated "Prisoners' Dilemma" game. However, this game does not seem directly applicable to information exchanging devices. Moreover, the chief result, while sensible, may seem trivial: the most effective strategy to achieve robust cooperation appears to be tit for tat, i.e. cooperate with agents that reciprocate the cooperation, stop cooperating with those that do not. More recent, tag-based models (e.g. [15] 7] start from a simpler situation than the Prisoners' Dilemma, in which one agent "donates" a service to another one, at a small cost to the donor but a larger benefit to the recipient. The main idea is that agents are identified by "tags", and that they cooperate with those agents whose tags are similar to their own. The rationale is that agents with the same type of tag belong to the same group, "family" or "culture", following the same rules, so that they can be trusted to reciprocate.

For artifacts, a tag may include such markers as brand, model, and proto-

cols understood. This would show that a device is capable and willing to lend particular services to another one, thus obviating the need for a repeated, "titfor-tat-like" interaction probing the willingness to reciprocate. Yet extensible environments should allow the addition of very dissimilar devices, made by different companies using different standards and functionalities. Therefore, we propose a different approach, combining some advantages of tags and tit-for-tat strategies.

Consider a game with the following moves: an agent makes a request and the other agent either cooperates (donates) or "defects". Agents learn from these interactions in the following manner: if the result is positive (cooperation), the agent will get more "trust" in the other agent's cooperativeness. Thus, the probability increases that it will make further requests to that agent in the future, 
or react positively to the other's requests. Vice-versa, a negative result will lead to more "distrust" and a reduced probability to make or accept requests to/from this agent. Still, to recognise this agent, it has to take its clue from the tag, which is usually not unique to that agent. This means that a later interaction may be initiated with a different agent that carries a similar tag, but that is not necessarily willing to cooperate to the same extent. We may assume that if the first few interactions with agents having similar tags all generate positive (negative) results, the agent will develop a default propensity to react positively (negatively) always to agents characterised by that type of markers.

We expect that in this way the initially undirected interactions will produce a differentiation in clusters of similarly marked agents that cooperate with each other (e.g. all devices belonging to the same user or organization), but that are reluctant to interact with members of other groups (e.g. devices belonging to rival organizations). The tags and their association thus develop the function of a mediator 9 that increases the probability of positive interactions by creating a division between "friends" (in-group) and "strangers" or "foes" (out-group). Note, however, that there is no assumption that an agent only cooperates with agents bearing the same tag as itself: by default it cooperates with anyone having a tag similar to the one of agents that were cooperative in the past. This means that there can be groups with which everyone cooperates (e.g. "public" devices), but also that specific types of "symbiosis" can develop in which one group systematically seeks out members of a different group to cooperate with because of their complementary capabilities. This brings us to the more complex issue of the division of labour.

\subsection{Learning to coordinate}

After having ascertained that our devices can communicate and cooperate, we still need to make sure that the functions they perform satisfy the user. This desired functionality can be viewed as a complex of tasks that need to be executed. The tasks are mutually dependent in the sense that a certain task (e.g. locating a file) has to be accomplished before subsequent tasks (e.g. downloading and playing the file) can be initiated. Each agent can either execute a task itself, or delegate it to another agent. Initially, we may assume that all agents that have a certain functionality built in (e.g. playing a sound file) are equally competent at performing that type of task. However, in practice the satisfaction of the user can vary. For example, a recording is likely to be played with a higher sound quality by a music installation than by a PDA or television. By default, devices can use certain preprogramed rules-of-thumb to decide who takes precedence (e.g. newer or more specialized devices are preferred to older, less specialized ones). Yet in an open environment there is no guarantee that such simple heuristics will produce the best result. Again, we may tackle this problem through individual learning coupled with collective self-organization.

Assume that the user regularly expresses his/her overall satisfaction with the ambient intelligence environment (e.g. explicitly by clicking on a scale 
from one to ten, or implicitly by facial or physiological cues that express happiness/unhappiness). This score can be used as a feedback signal to the network of devices, allowing it to reinforce the more successful rules, while weakening the less effective ones. We will assume that the agent who delegated a task will increase its trust in the competence of the agent that performed that task, and thus increase its probability to delegate a similar task to the same agent in the future. Otherwise, it will reduce its trust. As demonstrated by the simulation of Gaines [5], this assumption is sufficient to evolve a self-reinforcing division of labour where tasks are delegated to the most "expert" agents.

However, when the tasks are mutually dependent, selecting the right specialist to carry out a task is not sufficient: First the preparatory tasks have to be done by the right agents, in the right order. When the agents do not know a priori what the right order is, they can randomly attempt to execute or delegate a task, and, if this fails, pick out another task. Eventually they will find a task they can execute, either because it requires no preparation, or because a preparatory task has already been accomplished by another agent. Each completed task enables the accomplishment of a series of directly dependent tasks. In this way the overall problem will eventually be solved. In each problem cycle, agents will learn better when to take on which task by themselves, or when to delegate it to a specific other agent.

We expect that this learned organisation will eventually stabilise into a system of efficient, coordinated actions, adapted to the task structure. When new devices are added to the system, system and device should mutually adapt, producing a new organization. While no single agent knows how to tackle the entire problem, the knowledge has been "distributed" across the system. The "tags" that identify agents, and the learned associations between a tag and the competence for a particular task, play the role of a mediator 9], delegating tasks to the right agents and coordinating their interactions so that the problem is tackled as efficiently as possible.

\subsection{Conclusions}

We cannot keep on adding functions to personal computers. They serve as text editors, game consoles, televisions, home cinemas, radios, agendas, music players, gateway to the Internet, etc. Such general devices will never produce the same quality as specialized appliances. Our PCs are like ducks: they can swim, but not as well as fish; fly, but not as well as hawks; and walk, but not as well as cats. Rather than integrate so many functions in a single device, it seems preferable to entrust them to an ever expanding network of specialized devices that is kept coordinated through an ongoing process of self-organization. We have described a number of general requirements and approaches that may enable our artifacts to learn the most effective way of cooperation.

In our overall scenario, we have assumed that standard functions and interaction rules are preprogrammed by a global community to handle the most

common, default situations, but that the system is moreover ready to extend 
its own capabilities, adapting to newly encountered tasks, situations, or devices. This ability to adapt should be already present in the interaction rules. The adaptation may be achieved through the self-organization of the system of agents, using recurrent, "game-like" interactions, in which the agents learn

what messages mean and who they can trust to perform which task. Most of this can happen outside of, or in parallel with, their normal "work", using idle processing power to explore many different communication and collaboration configurations. Thus, we can imagine that our future, intelligent devices, like young animals or children, will learn to become more skilful by exploring, "playing games" with each other, and practising uncommon routines, so as to be prepared whenever the need for this kind of coordinated action appears.

\subsection{Acknowledgements}

We thank Peter McBurney for useful comments. C. G. was supported in part by CONACyT of Mexico.

\section{Bibliography}

[1] Axelrod, R. M., The Evolution of Cooperation, Basic Books New York (1984).

[2] Belpaeme, Tony, "Reaching coherent colour categories through communication", Proc. 13th Belgium-Netherlands Conference on AI (B. K. ET AL. ed.), (2001), 41-48.

[3] Bonabeau, E, M. Dorigo, and G. Theraulaz, Swarm Intelligence, Oxford University Press (1998).

[4] Dastani, Mehdi, Joris Hulstijn, and Leendert Van der Torre, "Negotiation protocols and dialogue games", International Conference on $\mathrm{Au}$ tonomous Agents, ACM (2001), $180-181$.

[5] Gaines, B. R., "The collective stance in modeling expertise in individuals and organizations", Int. J. Expert Systems 71 (1994), 22-51.

[6] Gershenson, Carlos, and Francis Heylighen, "When can we call a system self-organizing?", Advances in Artificial Life, 7th European Conference, ECAL 2003 LNAI 2801 (W. Banzhaf, T. Christaller, P. Dittrich, J. T. Kim, AND J. ZIEGLER eds.), Springer-Verlag (2003), 606-614.

[7] Hales, D., and B. Edmonds, "Evolving social rationality for MAS using "tags"", Proceedings of the 2nd International Conference on Autonomous Agents and Multiagent Systems (J. S. R. ET Al. ed.), ACM Press (2003), 497-503. 
[8] Heylighen, Francis, "Collective intelligence and its implementation on the web", Computational and Mathematical Theory of Organizations 5 (1999), 253-280.

[9] Heylighen, Francis, "Mediator evolution", Tech. Rep. no., Principia Cybernetica, (2003).

[10] Heylighen, Francis, and Carlos Gershenson, "The meaning of selforganization in computing", IEEE Intelligent Systems (July/August 2003), $72-75$.

[11] Hutchins, E, Cognition in the Wild, MIT Press (1995).

[12] Hutchins, E., and B. Hazelhurst, "How to invent a lexicon: The development of shared symbols in interaction", Artificial Societies (N. GILBERT AND R. Conte eds.), UCL Press (1995).

[13] ISTAG, "Scenarios for ambient intelligence in 2010", Tech. Rep. no., ISTAG, (2001).

[14] Reed, C., T. J. Norman, and N. R. Jennings, "Negotiating the semantics of agent communication languages", Computational Intelligence 18, 2 (2001), 229-252.

[15] Riolo, R., M. D. Cohen, and R. M. Axelrod, "Evolution of cooperation without reciprocity", Nature 414 (2001), 441-443.

[16] Steels, Luc, "Synthesising the origins of language and meaning using co-evolution, self-organisation and level formation", Approaches to the Evolution of Language (J. R. Hurford, M. Studdert-Kennedy, And C. Knight eds.), Cambridge University Press (1998), 384-404.

[17] Weiser, M., "Some computer science problems in ubiquitous computing", Communications of the ACM (July 1997).

[18] Wittgenstein, Ludwig, Philosophical Investigations 3rd ed., Prentice Hall (1999). 